PROCEEDINGS OF THE

AMERICAN MATHEMATICAL SOCIETY

Volume 125, Number 6, June 1997, Pages 1873-1879

S $0002-9939(97) 03780-5$

\title{
TOPOLOGICAL ENTROPY FOR GEODESIC FLOWS UNDER A RICCI CURVATURE CONDITION
}

\author{
SEONG-HUN PAENG
}

(Communicated by Mary Rees)

\begin{abstract}
It is known that the topological entropy for the geodesic flow on a Riemannian manifold $M$ is bounded if the absolute value of sectional curvature $\left|K_{M}\right|$ is bounded. We replace this condition by the condition of Ricci curvature and injectivity radius.
\end{abstract}

\section{INTRODUCTION}

The topological entropy is the most important invariant related to the orbit growth of a dynamical system. It represents the exponential growth rate for the number of orbit segments. In a sense, topological entropy describes the total exponential complexity of the orbit structure.

The topological entropy for geodesic flows is closely related to the curvatures of manifolds since geodesic flows depend on the metrics of manifolds. Let $\phi_{t}$ be a geodesic flow on a Riemannian manifold $M$ and $h\left(\phi_{t}\right)$ be the topological entropy for $\phi_{t}$. It was known that if the absolute value of sectional curvature satisfies $\left|K_{M}\right|<k$, then the topological entropy for geodesic flows satisfies $h\left(\phi_{t}\right) \leq(n-1) \sqrt{k}$ by [Ma2]. Manning also proved that in the case of $K_{M}<0$, the topological entropy for geodesic flows is the same as the volume growth rate, $\lim _{r \rightarrow \infty} r^{-1} \log V(x, r)$, where $V(x, r)$ is the volume of the ball $B(x, r)$ contained in the universal covering space [Ma1]. This result was extended to the case of manifolds without conjugate points by Mañé $[\mathrm{FM}]$.

Bishop's comparison theorem [GHL] implies that if Ricci curvature satisfies $\operatorname{Ric}_{M} \geq-k$ and the injectivity radius of the universal covering space of $M$ is infinite, then $h\left(\phi_{t}\right) \leq \sqrt{(n-1) k}$.

We prove the following theorem;

Theorem. Let $k, i_{0}$ be positive real numbers. Then there exists a constant $C\left(i_{0}, n, k\right)$ such that for every $n$-dimensional compact Riemannian manifold $M$ with $\operatorname{Ric}_{M} \geq$ $-k$, $\operatorname{inj}_{M} \geq i_{0}$, the topological entropy for geodesic flow of $M$ is bounded by $C\left(i_{0}, n, k\right)$, where $\operatorname{inj}_{M}$ is the injectivity radius of $M$.

Received by the editors August 23, 1995 and, in revised form, October 17, 1995 and December 21, 1995.

1991 Mathematics Subject Classification. Primary 58F17; Secondary 53C20, 53C21, 53C22.

Partially supported by the Basic Science Research Institute Program and in part supported by GARC-KOSEF.

(C) 1997 American Mathematical Society 
For the proof, we will use Brocks' estimate for the Laplacian of the distance function, which will play an important role on estimating the norm of Jacobi fields.

We would like to express our gratitude to Professor Hongjong Kim and Professor Dong-Pyo Chi for much kind and helpful advice.

\section{Preliminaries}

Let $X$ be a compact metric space with the distance function $d$, and let $\phi=$ $\left\{\phi_{t}: X \rightarrow X\right\}$ be a flow, i.e. 1-parameter subgroup of homeomorphisms.

Define

$$
d_{T}^{\phi}(x, y):=\max \left\{d\left(\phi_{t}(x), \phi_{t}(y)\right) \mid 0 \leq t \leq T\right\} .
$$

Let $S_{d}(\phi, \epsilon, T)$ be the minimal number of balls of radius $\epsilon$ in the metric $d_{T}^{\phi}$ which cover $X$. Define

$$
h_{d}(\phi, \epsilon):=\lim \sup _{T \rightarrow \infty} \frac{1}{T} \log S_{d}(\phi, \epsilon, T) .
$$

Then the topological entropy for $\phi$ is defined as follows:

$$
h(\phi):=\lim _{\epsilon \rightarrow 0} h_{d}(\phi, \epsilon) .
$$

The following proposition is Brocks' estimate on the Laplacian of the distance function.

Proposition ([B, DSW]). Let $M$ be an n-dimensional complete Riemannian manifold with $\operatorname{Ric}_{M} \geq-k, \operatorname{inj}_{M} \geq i_{0}$. Let $r$ be a distance function from $p$ and $\gamma$ be a geodesic from $p$. Consider $r$ and $\triangle r$ as functions of $t$ on $\gamma$. Then $C_{1}\left(i_{0}, n, k\right) \leq$ $\triangle r-\frac{n-1}{t} \leq C_{2}\left(i_{0}, n, k\right)$ for some constants $C_{1}, C_{2}$ depending only on $i_{0}, n, k$ on $\left[0, i_{0} / 2\right]$.

The following lemma is important to estimate the upper bound of the topological entropy. It is proved in the discrete time case in $[\mathrm{KH}]$. In this case, almost the same proof can be applied.

Lemma 1. Let $X$ be a compact metric space and $\phi=\left\{\phi_{t}\right\}$ be a flow on $M$ with $d\left(\phi_{t}(x), \phi_{t}(y)\right) \leq e^{t C} d(x, y)$ for some constant $C$. Then $h(\phi) \leq|C| D(X)$ where $D(X)$ is the ball dimension defined by $D(X):=\lim _{\epsilon \rightarrow 0} \frac{\log b(\epsilon)}{|\log (\epsilon)|}$, and $b(\epsilon)$ is the minimum number of a covering of $X$ by $\epsilon$-balls.

Proof. By assumption, $d\left(\phi_{t}(x), \phi_{t}(y)\right) \leq e^{T|C|} d(x, y)$, for $t \leq T$. Then we easily know $\phi_{t}\left(B\left(x, \epsilon / e^{T|C|}\right)\right) \subset B\left(\phi_{t}(x), \epsilon\right)$, for $t \leq T$. So $B_{d}\left(x, \epsilon / e^{T|C|}\right) \subset B_{d_{T}^{\phi}}(x, \epsilon)$ and $S(\phi, \epsilon, t) \leq b\left(\epsilon / e^{T|C|}\right)$, where $B_{d}(x, r)$ is the $r$-ball in $d$-metric.

Then we obtain

$$
\begin{aligned}
\lim \sup _{T \rightarrow \infty} \frac{1}{T} \log S(\phi, \epsilon, T) & \leq \lim \sup _{T \rightarrow \infty} \frac{1}{T} \log b\left(\epsilon / e^{T|C|}\right) \\
& \leq \lim \sup _{T \rightarrow \infty} \frac{1}{T} \frac{\log b\left(\epsilon / e^{T|C|}\right)}{\log \left(\epsilon / e^{T|C|}\right)}\left|\log \left(\epsilon / e^{T|C|}\right)\right| \\
& \leq D(X)|C| .
\end{aligned}
$$

It is known that $D(X)=\operatorname{dim} X$, when $X$ is a topological manifold. 


\section{Proof of Theorem}

In the calculation of the entropy, it is an important step to define a metric on the unit tangent bundle, $T_{1} M$. Let $\gamma_{v}(t)$ be the geodesic on $M$ with $\gamma_{v}^{\prime}(0)=v_{p}$.

In Manning's paper [Ma1], a metric on $T_{1} M$ is defined as follows:

$$
d_{1}\left(v_{p}, w_{q}\right):=\sup _{0 \leq t \leq 1} d\left(\gamma_{v}(t), \gamma_{w}(t)\right) .
$$

In $[\mathrm{KH}]$, they used the following metric:

$$
d_{2}\left(v_{p}, w_{q}\right):=d(p, q)+\left\|P_{p}^{q}\left(v_{p}\right)-w_{q}\right\|,
$$

where $P_{p}^{q}\left(v_{p}\right)$ is the parallel translation of $v_{p}$ along the geodesic from $p$ to $q$.

Now we construct a metric on $T_{1} M$ as follows. Since $\operatorname{inj}_{M} \geq i_{0}$, it is possible to identify $v_{p} \in T_{1} M$ with the geodesic $\gamma_{v}(t), t \in\left[0, i_{0} / 4\right]$ with $\gamma_{v}^{\prime}(0)=v_{p}$. Then we may consider a Jacobi field $J$ along $\gamma_{v}(t)$ as a tangent vector at $v_{p} \in T_{1} M$, where $J$ is generated by a $C^{\infty}$-rectangle $Q(t, s)$ such that $\frac{\partial Q}{\partial s}(t, 0)=J(t),\left\|\frac{\partial Q}{\partial t}(t, s)\right\|=1$ for any $s$ and $0 \leq t \leq i_{0} / 4$ and $Q\left(t, s_{0}\right)$ is a geodesic for any fixed $s_{0}$. In this identification, we consider a $C^{\infty}$-rectangle, $Q(t, s)$, where $0 \leq s \leq 1$ and $0 \leq t \leq$ $i_{0} / 4$, as a $C^{\infty}$-curve on $T_{1} M$ from $\frac{\partial Q}{\partial t}(0,0)$ to $\frac{\partial Q}{\partial t}(0,1)$.

Then we define an inner product of tangent vectors and a distance on $T_{1} M$ as follows.

Definition. Let $J_{1}, J_{2}$ be tangent vectors on $T_{1} M$, i.e. Jacobi fields with above property. Then

$$
\begin{gathered}
\left(J_{1}, J_{2}\right)=\int_{0}^{i_{0} / 4}\left\langle J_{1}(t), J_{2}(t)\right\rangle d t \\
d\left(v_{p}, w_{q}\right)=\inf \int_{0}^{1}\left(\frac{\partial Q}{\partial s}, \frac{\partial Q}{\partial s}\right)^{\frac{1}{2}} d s=\inf \int_{0}^{1} \int_{0}^{i_{0} / 4}\left\|\frac{\partial Q}{\partial s}\right\| d t d s,
\end{gathered}
$$

where the inf is taken over piecewise $C^{\infty}$-curves $Q$ on $T_{1} M$ from $v_{p}$ to $w_{q}$, i.e. $\frac{\partial Q}{\partial t}(0,0)=v_{p}$ and $\frac{\partial Q}{\partial t}(0,1)=w_{q}$.

Since $T_{1} M$ is a compact Hausdorff space, the above metrics induce the same topology. Now we will prove a key lemma.

Lemma 2. Let $M$ be a complete Riemannian manifold with $\operatorname{Ric}_{M} \geq-k$, inj ${ }_{M} \geq i_{0}$ and let $\gamma(t)$ be a minimal geodesic starting from $q$ and $J(t)$ is a Jacobi field along $\gamma$ such that $J(0)=0$ and $\left\langle J^{\prime}(0), \gamma^{\prime}(0)\right\rangle=0$. Then $\|J\|(t) \leq e^{D} t\left\|J^{\prime}\right\|(0)$ for some constant $D\left(i_{0}, n, k\right)$ if $t<i_{0} / 2$.

Proof. The first half of the proof of this lemma is contained in the proof of the proposition 5.1 of [DSW]. Let $v, w \in T_{q} M$ and $\|v\|=\|w\|=l_{0} \leq i_{0} / 2$. Define $Q(t, s):=\exp (t V(s))$, where $V(s)$ is the geodesic on $S^{n-1}$ such that $V(0)=$ $v, V\left(s_{0}\right)=w$. Let $\gamma(t)=\exp _{q} t V(0)$ and $r(x)=d(q, x)$. Then $J(t)=\frac{\partial Q}{\partial s}(t, 0)$ is a Jacobi field with above property. Then,

$$
J^{\prime}=\nabla_{\gamma^{\prime}} J=\nabla_{J} \gamma^{\prime}=\nabla_{J} \nabla r=\nabla \nabla r(J) .
$$

Define $A:=\nabla \nabla r=$ Hess $r$, so $\operatorname{tr} A=\triangle r$ and $J^{\prime}=A J$. 
Now we will estimate the $\|A\|$. Write $A(t)=B(t)+I / t$. From the Jacobi equation $J^{\prime \prime}+R(J, T) T=0$ and $J^{\prime}=A J$, we obtain the Riccati equation $A^{\prime}+$ $A^{2}+R=0$ and substituting $A(t)=B(t)+I / t$, we get $B^{\prime}+B^{2}+\frac{2}{t} B+R=0$. Then, $\operatorname{tr} B^{\prime}+\|B\|^{2}+\frac{2}{t} \operatorname{tr} B+\operatorname{Ric}\left(\gamma^{\prime}\right) \leq 0$ since $\|B\|^{2} \leq \operatorname{tr} B^{t} B=\operatorname{tr} B^{2}$, where $B^{t}$ is the transpose of $B$ and $B$ is a symmetric matrix.

Multiplying the above equality by a factor $t^{\frac{1}{2}}$ and integrating along $\gamma(t)$, we obtain the following equality:

$$
\int_{0}^{l_{0}}\|B\|^{2} t^{\frac{1}{2}} \leq-l_{0}^{\frac{1}{2}} \operatorname{tr} B\left(l_{0}\right)-\frac{3}{2} \int_{0}^{l_{0}} t^{-\frac{1}{2}} \operatorname{tr} B(t)-\int_{0}^{l_{0}} t^{\frac{1}{2}} \operatorname{Ric}\left(\gamma^{\prime}\right) .
$$

Also using the Proposition ([B, DSW] $)$ in $\S 2,\left|\triangle r-\frac{n-1}{t}\right| \leq C\left(i_{0}, n, k\right)$ for some constant $C\left(i_{0}, n, k\right)$ on $\left[0, i_{0} / 2\right]$. Then we get $|\operatorname{tr} B|=\left|\operatorname{tr} A-\frac{n-1}{t}\right|=\left|\triangle r-\frac{n-1}{t}\right| \leq$ $C\left(i_{0}, n, k\right)$. So $\int_{0}^{l_{0}}\|B\|^{2} t^{\frac{1}{2}} \leq D_{1}\left(i_{0}, n, k\right)$, for some constant $D_{1}$.

Using the Hölder inequality,

$$
\int_{0}^{l_{0}}\|B\| \leq\left(\int_{0}^{l_{0}} t^{-\frac{1}{2}}\right)^{\frac{1}{2}}\left(\int_{0}^{l_{0}} t^{\frac{1}{2}}\|B\|^{2}\right)^{\frac{1}{2}} \leq D\left(i_{0}, n, k\right) .
$$

Then we have

$$
\begin{gathered}
\|J\|^{\prime} \leq\left\|J^{\prime}\right\| \leq\left\|\left(B+\frac{I}{t}\right)(J)\right\| \leq\|B\|\|J\|+\frac{\|J\|}{t}, \\
\int_{\delta}^{l_{0}} \frac{\|J\|^{\prime}}{\|J\|} \leq D+\log l_{0}-\log \delta, \\
\log \left(\frac{\|J\|\left(l_{0}\right)}{\|J\|(\delta)}\right) \leq D+\log \left(\frac{l_{0}}{\delta}\right), \\
\|J\|\left(l_{0}\right) \leq e^{D} l_{0} \frac{\|J\|(\delta)}{\delta} \\
\|J\|\left(l_{0}\right) \leq \lim _{\delta \rightarrow 0} e^{D} l_{0} \frac{\|J\|(\delta)}{\delta}=e^{D} l_{0}\left\|J^{\prime}\right\|(0),
\end{gathered}
$$

which completes the proof.

From Lemma 5.2 in [DSW], we know that $\|J(t)\| \leq \frac{t}{t_{0}} e^{D}\left\|J\left(t_{0}\right)\right\|, \quad 0 \leq t \leq t_{0}<$ $i_{0}$. Then $\left\|J^{\prime}(0)\right\|=\lim _{t \rightarrow 0} \frac{\|J(t)\|}{t} \leq \frac{e^{D}\left\|J\left(t_{0}\right)\right\|}{t_{0}}$.

From lemma 2 and the above inequality, we obtain the following inequality:

$$
e^{-D}\left\|J^{\prime}(0)\right\| t \leq\|J(t)\| \leq e^{D}\left\|J^{\prime}(0)\right\| t .
$$

Also we know that

$$
K_{1}\left\|J\left(i_{0} / 4\right)\right\| \leq\left\|J^{\prime}(0)\right\| \leq K_{2}\left\|J\left(i_{0} / 4\right)\right\|,
$$

for some constants $K_{1}, K_{2}$ depending only on $i_{0}, n, k$. Consequently,

$$
K_{3}\left\|J\left(i_{0} / 4\right)\right\| t \leq\|J(t)\| \leq K_{4}\left\|J\left(i_{0} / 4\right)\right\| t
$$

If $\left\langle J^{\prime}(0), \gamma^{\prime}(0)\right\rangle \neq 0$, we can decompose $J$ into tangential and perpendicular components and obtain the above boundedness (3), since tangential component is linear in $t[\mathrm{DSW}]$. 
Lemma 3. If $h$ is sufficiently small,

$$
d\left(\phi_{h}\left(v_{p}\right), \phi_{h}\left(w_{q}\right)\right)-d\left(v_{p}, w_{q}\right) \leq N\left(i_{0}, n, k\right) d\left(v_{p}, w_{q}\right) h,
$$

for some constant $N\left(i_{0}, n, k\right)$.

Proof. Let $Q(t, s)$ be a length minimizing curve from $v_{p}$ to $w_{q}$, i.e. a piecewise $C^{\infty}$ rectangle which realizes the distance from $v_{p}$ to $w_{q}$. We may assume the existence of a length minimizing curve.

Then we know that

$$
d\left(v_{p}, w_{q}\right)=\int_{0}^{1} \int_{0}^{i_{0} / 4}\left\|\frac{\partial Q}{\partial S}\right\| d t d s
$$

and

$$
d\left(\phi_{h}\left(v_{p}\right), \phi_{h}\left(w_{q}\right)\right) \leq \int_{0}^{1} \int_{h}^{i_{0} / 4+h}\left\|\frac{\partial Q}{\partial s}\right\| d t d s
$$

So we get

$$
d\left(\phi_{h}\left(v_{p}\right), \phi_{h}\left(w_{q}\right)\right)-d\left(v_{p}, w_{q}\right) \leq \int_{0}^{1} \int_{0}^{h}+\int_{0}^{1} \int_{i_{0} / 4}^{i_{0} / 4+h}\left\|\frac{\partial Q}{\partial s}\right\| d t d s .
$$

Let $\frac{\partial Q}{\partial s}(0, s)=V(s)$ and $\frac{\partial Q}{\partial s}\left(i_{0} / 4, s\right)=W(s)$. Decompose $J$ into $J_{1}$ and $J_{2}$ such that $J_{1}(0)=0, J_{2}\left(i_{0} / 4\right)=0$. Then $\left\|J_{2}(0)\right\|=\|V\|$ and $\left\|J_{1}\left(i_{0} / 4\right)\right\|=\|W\|$. If $\|V\|=\|W\|=0$, then $J(t)=J_{1}(t)=J_{2}(t)=0$ for all $t \leq i_{0} / 4$ and $v_{p}=w_{q}$. So we do not need consider this case. From now on we may assume $\|V\| \neq 0$. Then by (3), we have

$$
\begin{gathered}
K_{3}\|W\| t \leq\left\|J_{1}(t)\right\| \leq K_{4}\|W\| t, \\
K_{3}\|V\| t \leq\left\|J_{2}\left(i_{0} / 4-t\right)\right\| \leq K_{4}\|V\| t .
\end{gathered}
$$

Thus we have

$$
\begin{aligned}
d\left(\phi_{h}\left(v_{p}\right), \phi_{h}\left(w_{q}\right)\right)-d\left(v_{p}, w_{q}\right) & \leq \int_{0}^{h} \int_{0}^{1}+\int_{i_{0} / 4}^{i_{0} / 4+h} \int_{0}^{1}\|J(t)\| d s d t \\
& \leq \int_{0}^{h} \int_{0}^{1}+\int_{i_{0} / 4}^{i_{0} / 4+h} \int_{0}^{1}\left\|J_{1}(t)\right\| d s d t \\
& +\int_{0}^{h} \int_{0}^{1}+\int_{i_{0} / 4}^{i_{0} / 4+h} \int_{0}^{1}\left\|J_{2}(t)\right\| d s d t \\
& \leq\left(\int_{i_{0} / 4}^{i_{0} / 4+h}+\int_{0}^{h} K_{4} t d t\right)\left(\int_{0}^{1}\|V\|+\|W\| d s\right) \\
& \leq K\left(i_{0}, n, k\right)\left(\int_{0}^{1}\|V\|+\|W\| d s\right) h,
\end{aligned}
$$

for some constant $K\left(i_{0}, n, k\right)$. Now compute the $d\left(v_{p}, w_{q}\right)$. Let

$$
a=\frac{i_{0} K_{3}\|V\|}{4\left(K_{3}\|V\|+K_{4}\|W\|\right)}, \quad b=\frac{i_{0} K_{3}\|W\|}{4\left(K_{3}\|W\|+K_{4}\|V\|\right)} .
$$


On $[0, a]$, we know that $\left\|J_{2}(t)\right\| \geq\left\|J_{1}(t)\right\|$ and on $\left[i_{0} / 4-b, i_{0} / 4\right],\left\|J_{1}(t)\right\| \geq\left\|J_{2}(t)\right\|$ by (4) and (5). So we obtain

$$
\begin{aligned}
d\left(v_{p}, w_{q}\right) & =\int_{0}^{i_{0} / 4} \int_{0}^{1}\|J\| d s d t \\
& \geq \int_{0}^{1} \int_{0}^{a}\left\|J_{2}\right\|-\left\|J_{1}\right\| d t d s+\int_{0}^{1} \int_{i_{0} / 4-b}^{i_{0} / 4}\left\|J_{1}\right\|-\left\|J_{2}\right\| d t d s \\
& \geq \int_{0}^{1} \int_{0}^{a} K_{3}\|V\|\left(i_{0} / 4-t\right)-K_{4}\|W\| t d t d s \\
& +\int_{0}^{1} \int_{i_{0} / 4-b}^{i_{0} / 4} K_{3}\|W\| t-K_{4}\|V\|\left(i_{0} / 4-t\right) d t d s
\end{aligned}
$$

The integral $\int_{0}^{a} K_{3}\|V\|\left(i_{0} / 4-t\right)-K_{4}\|W\| t d t$ is the area of

$$
\left\{(x, y) \mid K_{4}\|W\| x \leq y \leq K_{3}\|V\|\left(i_{0} / 4-x\right), \quad x \leq a\right\} .
$$

Hence we get

$$
\int_{0}^{a}\left\|J_{2}\right\|-\left\|J_{1}\right\| \geq a i_{0} K_{3}\|V\| / 8=i_{0}^{2} K_{3} / 32 \frac{K_{3}\|V\|^{2}}{K_{4}\|W\|+K_{3}\|V\|} .
$$

Similarly we get

$$
\int_{i_{0} / 4-b}^{i_{0} / 4}\left\|J_{1}\right\|-\left\|J_{2}\right\| \geq i_{0}^{2} K_{3} / 32 \frac{K_{3}\|W\|^{2}}{K_{4}\|V\|+K_{3}\|W\|} .
$$

It is an easy calculation that

$$
\int_{0}^{i_{0} / 4}\|J\| d t \geq \frac{a_{1}\left(\|V\|^{2}\|W\|+\|V\|\|\mid W\|^{2}\right)+a_{2}\left(\|V\|^{3}+\|W\|^{3}\right)}{b_{1}\left(\|W\|^{2}+\|V\|^{2}\right)+b_{2} \mid\|V\|\|W\|},
$$

for some positive constant $a_{i}, b_{i}$ depending only on $i_{0}, n, k$.

Then we obtain

$$
\begin{aligned}
\frac{\|V\|+\|W\|}{\int_{0}^{i_{0} / 4}\|J\| d t} & \leq \frac{(\|V\|+\|W\|)\left(b_{1}\left(\|V\|^{2}+\|W\|^{2}\right)+b_{2}\|V\| \mid\|W\|\right)}{a_{1}\left(\|V\|^{2}\|W\|+\|V\| \mid\|W\|^{2}\right)+a_{2}\left(\|V\|^{3}+\|W\|^{3}\right)} \\
& =\frac{b_{1}\left(\|V\|^{3}+\|W\|^{3}\right)+c_{1}\left(\|V\|^{2}\|W\|+\|V\|\|W\|^{2}\right)}{a_{2}\left(\|V\|^{3}+\|W\|^{3}\right)+a_{1}\left(\|V\|^{2}\|W\|+\|V\|\|W\|^{2}\right)} \\
& =\frac{b_{1}\|V\|^{3}+c_{1}\|V\|^{2}\|W\|+c_{1}\|V\|\|W\|^{2}+b_{1}\|W\|^{3}}{a_{2}\|V\|^{3}+a_{1}\|V\|^{2}\|W\|+a_{1}\|V\|\|W\|^{2}+a_{2}\|W\|^{3}} \\
& =\frac{b_{1}+c_{1}\|W\| /\|V\|+c_{1}\|W\|^{2} /\|V\|^{2}+b_{1}\|W\|^{3} /\|V\|^{3}}{a_{2}+a_{1}\|W\| /\|V\|+a_{1}\|W\|^{2} /\|V\|^{2}+a_{2}\|W\|^{3} /\|V\|^{3}} \\
& \leq C_{0}\left(i_{0}, n, k\right),
\end{aligned}
$$

for some positive constants $c_{1}\left(i_{0}, n, k\right), C_{0}\left(i_{0}, n, k\right)$, since we assume $\|V\| \neq 0$ and we know the boundedness of $\frac{e_{0}+e_{1} x+e_{2} x^{2}+e_{3} x^{3}}{f_{0}+f_{1} x+f_{2} x^{2}+f_{3} x^{3}}$ from

$$
\lim _{x \rightarrow 0} \frac{e_{0}+e_{1} x+e_{2} x^{2}+e_{3} x^{3}}{f_{0}+f_{1} x+f_{2} x^{2}+f_{3} x^{3}}=\frac{e_{0}}{f_{0}}
$$


and

$$
\lim _{x \rightarrow \infty} \frac{e_{0}+e_{1} x+e_{2} x^{2}+e_{3} x^{3}}{f_{0}+f_{1} x+f_{2} x^{2}+f_{3} x^{3}}=\frac{e_{3}}{f_{3}}
$$

for positive constants $e_{i}, f_{i}$ and $x \geq 0$.

Consequently, we have

$$
\begin{aligned}
d\left(\phi_{h}\left(v_{p}\right), \phi_{h}\left(w_{q}\right)\right)-d\left(v_{p}, w_{q}\right) & \leq K\left(\int_{0}^{1}\|V\|+\|W\| d s\right) h \\
& \leq K C_{0}\left(\int_{0}^{1} \int_{0}^{i_{0} / 4}\|J\| d t d s\right) h \\
& =K C_{0} d\left(v_{p}, w_{q}\right) h,
\end{aligned}
$$

which completes the proof.

For fixed $T$, we have

$$
\begin{aligned}
d\left(\phi_{T}\left(v_{p}\right), \phi_{T}\left(w_{q}\right)\right) & \leq \lim _{h \rightarrow 0}\left(1+K C_{0} h\right)^{T / h} d\left(v_{p}, w_{q}\right) \\
& \leq e^{T K C_{0}} d\left(v_{p}, w_{q}\right) .
\end{aligned}
$$

Then by lemma 1, the topological entropy for geodesic flows of compact Riemannian manifolds with $\operatorname{Ric}_{M} \geq-k$ and $\operatorname{inj}_{M} \geq i_{0}$ is bounded; i.e. $h(\phi) \leq(2 n-1) K C_{0}$. This completes the proof of the theorem.

\section{REFERENCES}

[AC] M. Anderson, J. Cheeger, $C^{\alpha}$-compactness for manifolds with Ricci curvature and injectivity radius bounded below, J. Differential Geometry 35 (1992), 265-281. MR 93c:53028

[B] R. Brocks, Abstandsfunktion, Riccikrümmung und Injektivitätsradius, Diplomarbeit, University of Münster (1993).

[CE] J. Cheeger, D. G. Ebin, Comparison theorems in Riemannian geometry, Amsterdam, 1975. MR 56:16538

[DSW] X. Dai, Z. Shen, G. Wei, Negative Ricci curvature and isometry group, Duke Math. J. 76 (1994), 59-73. MR 96e:53051

[FM] A. Freire, R. Mañé, On the entropy of geodesic flow in manifolds without conjugate points, Invent. Math. 69 (1982), 375-392. MR 84d:58063

[GHL] S. Gallot, D. Hulin, J. Lafontaine, Riemannian geometry, Springer-Verlag, 1987. MR 88k:53001

$[\mathrm{H}] \quad$ J. Heo, Completely multi-positive linear maps and representations of Hilbert $C^{*}$ modules, preprint.

[KH] A. B. Katok, B. Hasselblatt, Introduction to the modern theory of dynamical systems, Cambridge University Press, 1995. MR 96c:58055

[Ma1] A. Manning, Topological entropy for geodesic flows, Ann. Math. 110 (1979), 567-573. MR 81e: 58044

[Ma2] More topological entropy for geodesic flows, Dynamical systems and turbulence, Warwick (1980), 243-250. MR 84j:58096

[P] S.-H. Paeng, A sphere theorem under a curvature perturbation, Kyushu J. Math. 50 (1996), 459-470.

Department of Mathematics, Seoul National University, Seoul 151-742, Korea

E-mail address: shpaeng@math.snu.ac.kr 